\title{
An adaptive container code character segmentation algorithm
}

\author{
Yajie Zhu ${ }^{1, a}$, Chenglong Liang ${ }^{2, b}$ \\ ${ }^{1}$ School of Information Engineering, Shanghai Maritime University, Shanghai 201306, China; \\ ${ }^{2}$ School of Transport \& Communications, Shanghai Maritime University, Shanghai 201306, China. \\ a769877925@qq.com, b554774134@qq.com
}

Keywords: adaptive, container code, character segmentation, morphology, projection.

\begin{abstract}
Container character segmentation is an important step of the container code intelligent recognition system, which greatly affects the subsequent character recognition work. In order to quickly segment a variety of container characters, an adaptive container character segmentation algorithm was proposed under the complex situation. The filtering operation was implemented on the extracted multiple code area; then it is determined whether that the container code is arranged in a row, a column or multiple rows according to the high-width ratio; finally, the character segmentation is performed on the filtered code images. First, the morphology dilation with the vertical linear structural elements and the left-right bound projection location were used to perform the segmentation task of the characters arranging in a row. Secondly, the morphological dilation with the horizontal structural elements and the upper-low bound projection location were used to perform the segmentation task of the characters arranging in a column. Finally, for the multiply row code characters image, it was divided into multiple character areas where each is in a row or column. Experimental results show that this method can accurately, quickly and adaptive segment the container character images in a row, a column or multiple rows under the complex background.
\end{abstract}

\section{Introduction}

In recent years, with the development of the transportation modernization, to simplify the container transport management, improve the efficiency of management, container code automatic identification technology has been more and more attention. The container of in and out needs to be tested at container terminals, customs, freight yard and other places, and the record of customs clearance of the container code is only by manual input, not only speed is slow, and error-prone, thus realize container code intelligent identification is very necessary. Container intelligent recognition system generally includes image processing, image acquisition, code area positioning, code character segmentation, code character recognition and so on several major parts, each part is closely linked. However, the code character segmentation is a key part of container intelligent identification system. the arrangement sort of the container code is more complex, can be horizontal, vertical, horizontal array can be divided into lines 1, 2, 3 and 4.Vertical alignment is usually 1 column. Therefore, container image under complex background, effectively remove interference, it is necessary that the method could quickly, accurately and adaptive character segmentation a variety of container code area.

\section{The theory of character segmentation}

(1) image processing

Step one, the image gray-scale transformation. Gray-scale transformation is due to container image is obtained by digital camera. It is full of color images before the image processing, and color image contains a large amount of information, this requires more storage space, therefore, the speed of the image processing will also slow. So the image gray-scale transformation can make the image contains only brightness information, color information is not included. The purpose is to improve the speed of the image processing and make it more convenient to calculate in the image 
processing.

Step two, contrast enhancement. Causes of gray image contrast enhancement is due to the captured images often have uneven illumination situation, coupled with the container box by dust pollution, brushing the top of the character will be affected, thereby reducing the contrast. Enhanced gray-scale image contrast is actually enhanced contrast portions of the image so that the color contrast is more obvious. In practice, it is to achieve by enhancing dynamic range of two gray values in the image.

Step three, the median filter. For reasons of image filtering is at the time of the container image processing, will encounter the effects of noise. The noise is a kind of damage to image information. Median filter is a nonlinear method of reducing, belongs to the space domain filtering method, it uses the adjacent pixel gray value of a pixel point instead of the gray value of the pixel, the purpose is in order to remove isolated noise points.

(2) Code type is determinate. Using the aspect ratio of the code area image after the processing to determine that code is one row, one column or more a row. If the aspect ratio of code area image is greater than 2 , then the code is a row, if the aspect ratio of code area image is less than 1, then the code is a column, otherwise the code is more a row.

(3) Character segmentation of one row code area

Step one, image binary. In this paper, the otsu method is used for binary images. Because the weather, shooting angle and the factors such as the oil box will affect to collect the container images, lead to the quality of collecting many container images is low. Some images will be serious uneven illumination and low contrast and edge character is not obvious. Otsu method is compared traditional paper methods to better meet the requirements of the binary image in this paper.

Step two, code color judgment. Through get the binary image after image processing, through the number of 1 and 0 of the container code binary image to discriminate code color. If the number of 1 is less than the number of 0 , the container code is white, otherwise, the container code is black and anti-black color, the black code transform into a white code so that code area will be character segmentation.

Step three, deleting the long vertical lines and small area. By statistics the number of code area's each column 1 and the height of the characters to determine the long vertical lines. If the number of the code area's column 1 is greater than the height of the characters, the long vertical lines and small area are removed, or the smaller area is deleted.

Step four, morphological dilation operation. When choosing structural elements, vertical linear structural element with the aspect ratio of one row code area is choice. The purpose is correctly to make that the code area characters are formed the connected area. With the structural elements to dilation operations for code area, in order to more convenient location the left and right border of each character of code area.

Step five, character segmentation. We use the pixel projection region segmentation method to locate the left and right boundary of each code character. Projection is a kind of characteristic function, it is a simplified two one-dimensional function of the two-dimensional pixel distribution image on $X$ and $Y$ axis, and projection algorithm is the departure from the two one-dimensional function to split [1].

Step six, character tilt correction. The tilt character is rotated counterclockwise, every rotates once to get the character aspect ratio, if the aspect ratio of the character is within the range of threshold, the rotating is stopped and character tilt correction is successful.

Step seven, fine character segmentation. Because the upper and lower boundary of the characters there are still some spare area, so you also need to be further divided, namely remove upper and lower boundary spare area of the characters. Fine segmentation algorithm is as follows: assuming that the size of the character images $f(x, y)$ as $M \times N$; Statistics the number on the white pixels each row of character image and placed a one-dimensional array $1 \times N$, positioning the position that the number is not zero in the first white pixel of the array from left to right, it is the upper boundary of the characters. Positioning the position that the number is not zero in the first white pixel of the array from right to left, it is the lower boundary of the characters; According to the upper and lower 
boundary of the character for the characters segmentation;

Step eight, character normalization. Because the height and width of the characters are different, so also they need to be the size of normalization [2] process. Code characters normalization is to adjust the size of those character images for the same size, the code characters are normalized to the same lattice character images. In this paper, the bilinear interpolation method for dividing and extracting code character image is normalized to the size $36 \times 20$ of the character [4].

Step nine, character stitching. The multiple normalized code characters are spliced together to form a complete picture by merging the matrix, so that a plurality of segmented characters are displayed in the overall image.

(4) one column code area character segmentation

The same as the one row code area character segmentation method.

(5) multi-line code area character segmentation

Step one, the image background elimination. The reason for this process is due to the captured image often have uneven illumination situation, combined with the container housing by dust pollution, brushing the top of the character will be affected, thereby reducing the contrast. Because this article taken the container image which the distance between the container image shot on the camera with the container 3 to $4 \mathrm{~m}$, so the container background eliminate methods in this paper as follows: (1) choose the size is $6 * 6$ square structural elements to get background image by the operation; (2) with original image subtracting the background image that is obtained the elimination of the background image.

Step two, image binary. The same as the one row code area character segmentation method.

Step three, code color judgment. The same as the one row code area character segmentation method.

Step four, multiply row code area segmentation algorithm: assuming that the size of the code area image $f(x, y)$ as $M \times N$; Statistics the number on the white pixels each row of code area image and placed a one-dimensional array $1 \times N$, positioning the position that the number is not zero in the first white pixel of the array from left to right, it is the upper boundary of the code area image. Positioning the position that the number is zero in the first white pixel of the array from left to right, it is the segmentation line of the code area image. Positioning the position that the number is not zero in the first white pixel of the array from left to right, it is the lower boundary of the code area image; According to the upper, segmentation line and lower boundary of the code area image for the code area image segmentation.

Step five, using a row code area image character segmentation method for multiple row code area image character segmentation.

\section{3. experimental results and analysis}

In this paper, all the experiments to be carried out in MATLAB R2012b.To a variety of code area image of extraction adaptive character segmentation study in this thesis, an adaptive container character segmentation algorithm was proposed under the complex situation.In this paper, the performance that adaptive container code locating under the complex situation is shown in figure. 


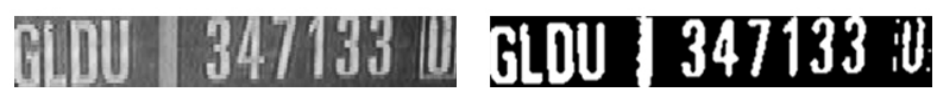

(a1) code area original image

FLOU: 347133 id FLOU 347133 V

(a3) delete long vertical line (a4) delete small area

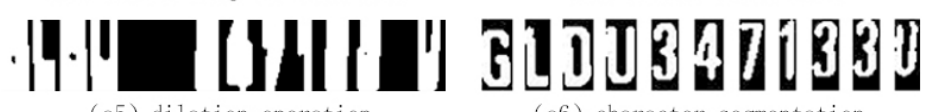

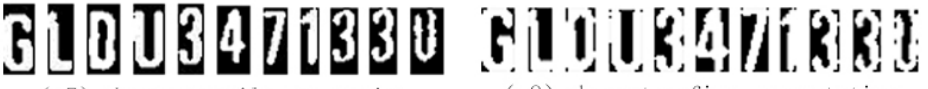

(a7) character tilt correction (a8) character fine segmentation

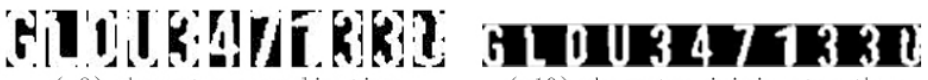

Figure 1 a row and white code area character segmentation process

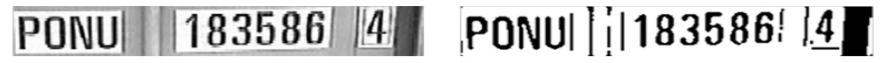

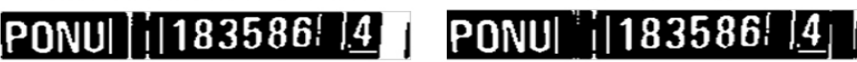

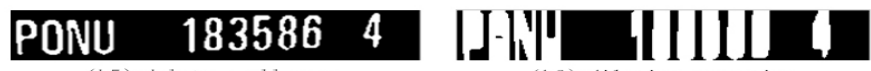

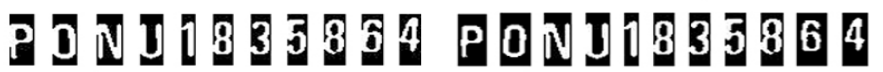

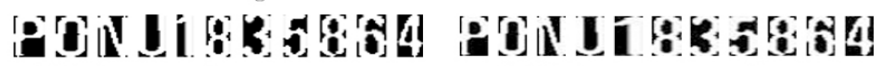

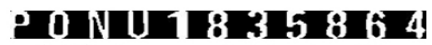

Figure 2 a row and black code area character segmentation process

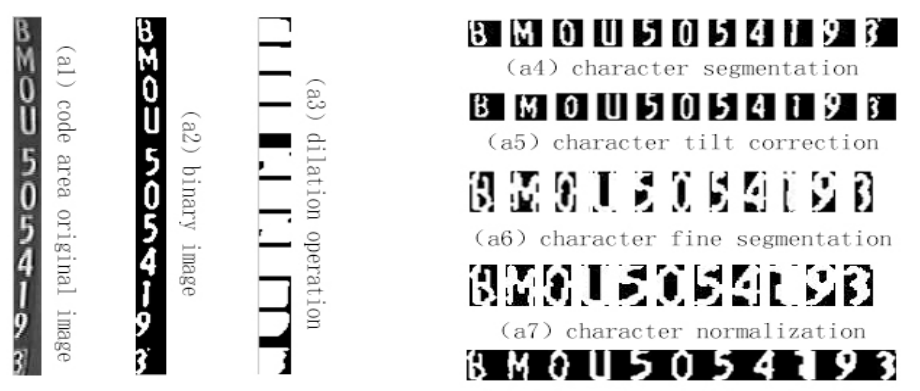

Figure 3 a column and white code area character segmentation process

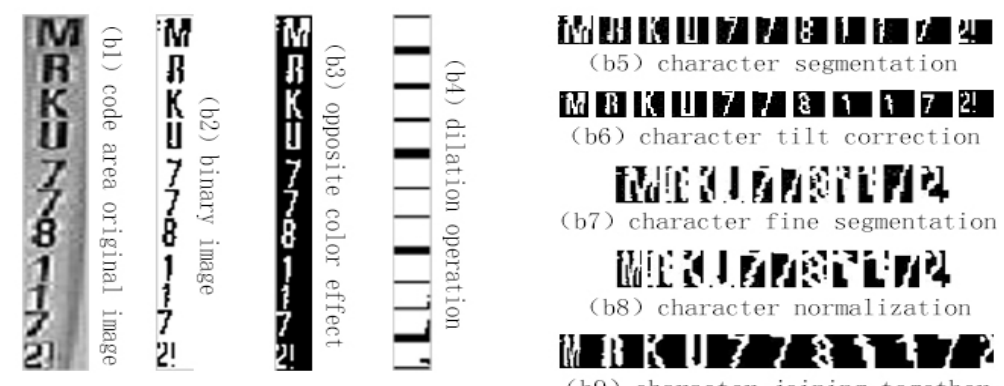

Figure 4 a column and black code area character segmentation process 


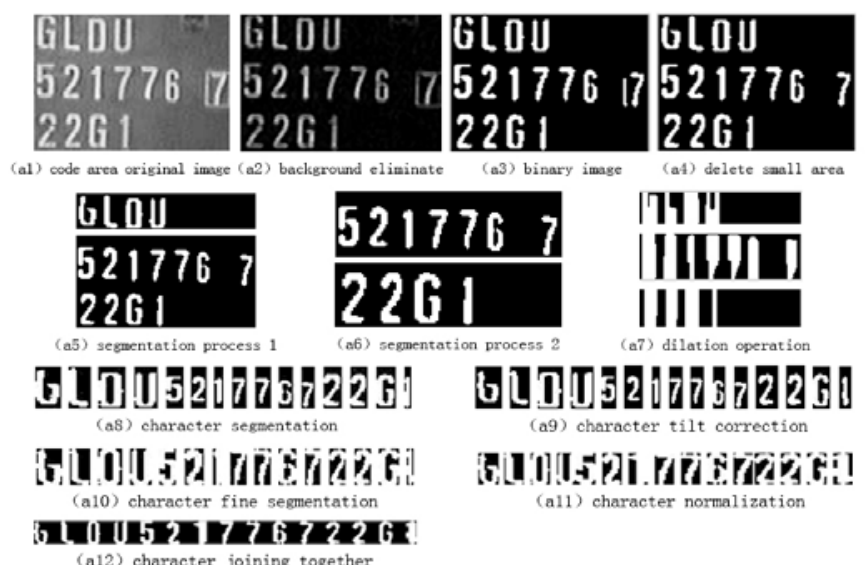

Figure 5 multiple row and white code area character segmentation process

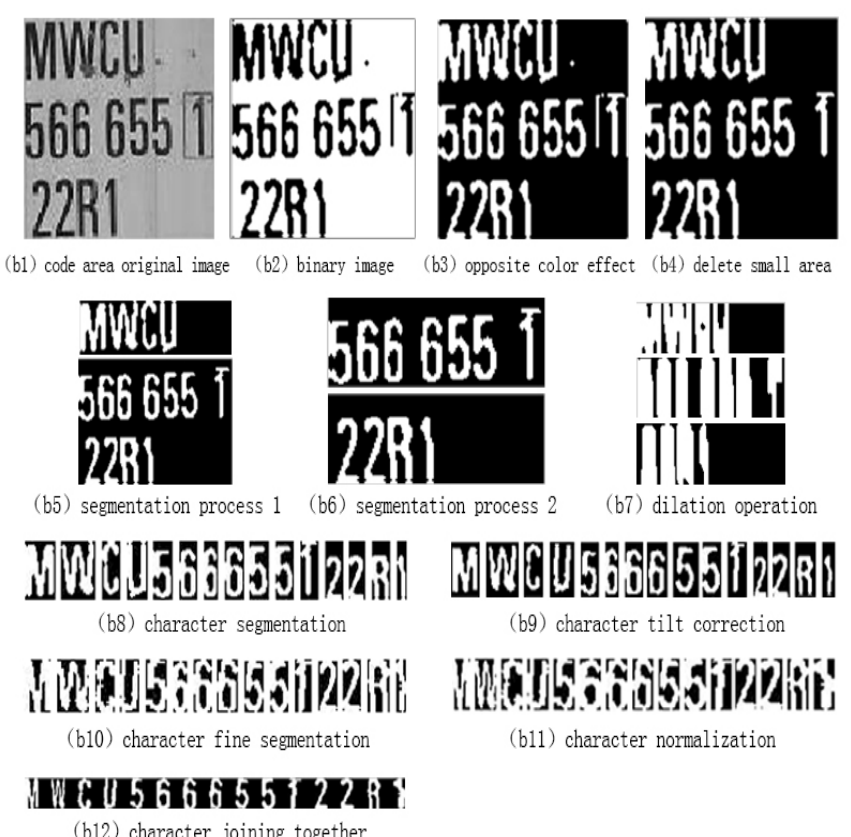

Figure 6 multiple row and black code area character segmentation process

\section{Summary}

Container logistics management computerization is the trend of The Times, this paper introduces the container code adaptive character segmentation algorithm, discusses the code area character segmentation of one row, multi-line and one column, it can be used for container intelligent identification system. This algorithm achieved good effect in practice, and is simple and practical, high efficiency.

\section{References}

[1] Xiaojuan Yang, Kai Song. Algorithm of document image segmentation based on projection method [J]. Journal of Chengdu University (Natural Science Edition), 2009, 28 (2): 139-141.

[2] Ting Hu. A Research of container character recognition based on neural network [D].Wuhan: Wuhan University of Technology, 2012.

[3] Linghong Sun. Study of container number smart recognition algorithm [D].Wuhan: Wuhan University of Technology, 2012. 
[4] B Shan. License plate character segmentation and recognition based on RBF neu- ral network $[\mathrm{C}] /$ Education Technology and Computer Science (ETCS), 2010 Sec- ond International Workshop on. IEEE, 2010: 86-89.

[5] S C Tai, T Y Chang, S Y Wang. A method of container codes detection and seg- mentation by using improved projection algorithm[C]//Intelligent Control and A- utomation (WCICA),2011 9th World Congress on. IEEE, 2011: 587-592. 